\title{
Neurocardiogenic Syncope during a Routine Colonoscopy: An Uncommon Malignant Presentation
}

\author{
Gautam Sharma, Nagendra Boopathy Senguttuvan, Rajnish Juneja and Vinay Kumar Bahl
}

\begin{abstract}
Neurocardiogenic syncope (NCS) is a common clinical entity. Most of these patients are managed medically by internists and general practitioners. Though NCS is frequently a benign disease, a malignant form of this disorder with episodes of prolonged cardioinhibition culminating in asystole is described. Here, we describe a 52-year-old woman who had such a life threatening malignant form of arrhythmia during a routine colonoscopy and review the literature of similar cases.
\end{abstract}

Key words: neurocardiogenic syncope, colonoscopy, malignant form, sudden cardiac death

(Intern Med 51: 891-893, 2012)

(DOI: 10.2169/internalmedicine.51.6622)

\section{Introduction}

Neurocardiogenic syncope (NCS) is a very common clinical entity. Though it is frequently benign, a malignant form of this disorder with episodes of prolonged cardioinhibition has been reported. These episodes can culminate in asystole, and if not interrupted, may simulate a sudden cardiac deathlike episode. The incidence of neurocardiogenic syncope decreases as age increases. In individuals aged $<40$ and individuals aged $>75$, the incidence of NCS is $51 \%$ and $25 \%$, respectively (1). Colonoscopy is a common investigational tool performed by endoscopists. It is a routine screening tool for elderly individuals (2). It is considered to be a safe procedure.

\section{Case Report}

A 52-year-old woman was evaluated for repeated symptoms suggestive of neurocardiogenic syncope. Her clinical examination and baseline laboratory tests including echocardiography, 24 hour holter and exercise treadmill test were normal. A head up tilt table test revealed an unprovoked mixed positive response. Within 3 minutes of upright positioning, the patient had symptomatic bradycardia and hypotension with a $\mathrm{BP}$ of $60 \mathrm{mmHg}$ and heart rate of 30 per minute culminating in a sinus pause of 3 seconds. The epi- sode was terminated by leg raising, intravenous fluids and atropine. She was discharged on tablet atenolol $50 \mathrm{mg}$ once a day on which she achieved a resting heart rate of 68 beats per minute. Her symptoms improved transiently. On a follow-up visit, she was put on holter monitoring to assess the response to beta blocker therapy and asked to come the following day for analysis.

The patient meanwhile consulted her gastroenterologist for irritable bowel syndrome which she had also been suffering from. Considering her age and initial nonresponsiveness to medical therapy, a diagnostic colonoscopy was planned by her treating physician. The preparation for colonoscopy was routine and included polyethylene glycol enema a day prior to the procedure. After premedicating her with $2 \mathrm{mg}$ intravenous midazolam, colonoscopy was done while the patient was still on holter monitoring. Twenty minutes into the procedure when the colonoscopy (which was a normal study) was almost complete, she experienced a syncopal episode, characterized by absent pulse and blood pressure, which required $1.2 \mathrm{mg}$ of atropine and $1 \mathrm{~L}$ of normal saline during the resuscitation. The holter recording analyzed the next day, dramatically demonstrated sinus bradycardia culminating in sinus arrest (which lasted $62 \mathrm{sec}-$ onds) during the episode (Fig. 1) The patient regained her baseline rhythm within 2 minutes of this event. The patient received a Medtronic (Medtronic, Minneapolis, MN, USA) rate drop responsive pacemaker on which she is presently 


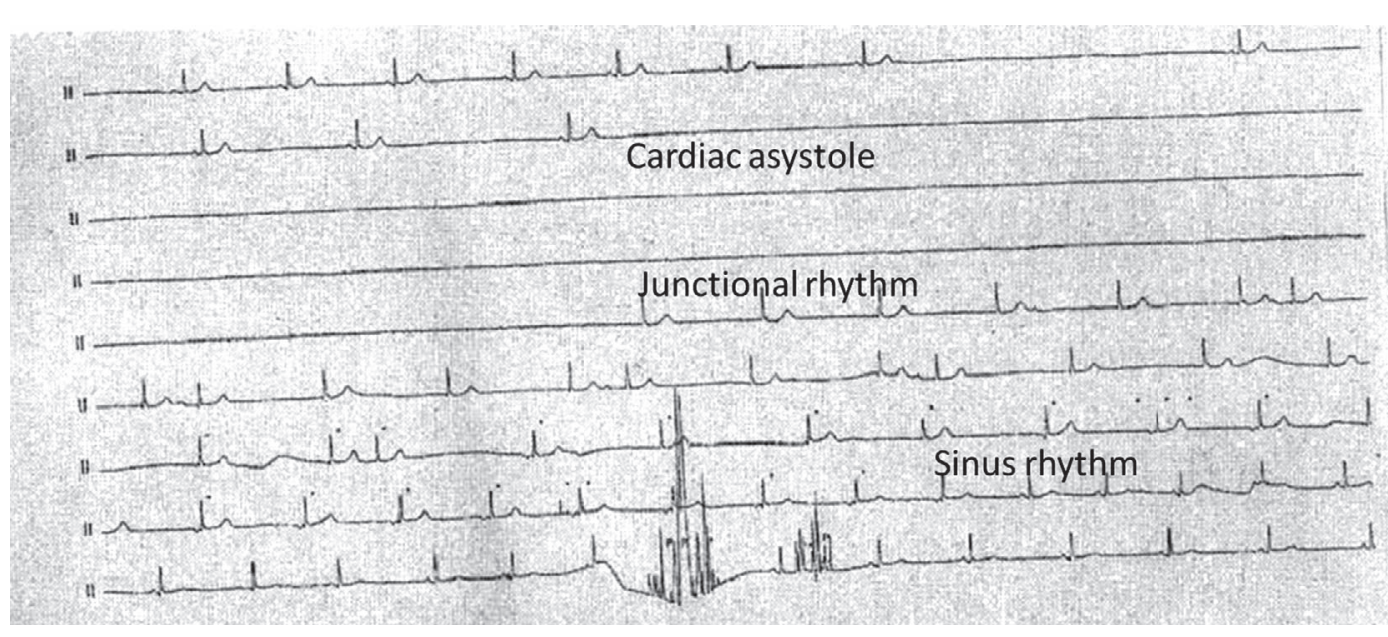

Figure 1. Single lead 24-hour ambulatory electrocardigram monitoring shows a prolonged cardiac asystole in lead II during colonoscopy. The episode started with sinus bradycardia and recovered with a junctional rhythm before culminating in sinus rhythm.

doing well. Pacemaker interrogation on subsequent followup, demonstrated numerous episodes of appropriate pacing during episodes of aborted cardioinhibitory syncope.

\section{Discussion}

Neurocardiogenic syncope (neurally mediated syncope) results from a vicious cycle of inappropriate peripheral vasodilatation leading to vigorous cardiac contractility that in turn stimulates afferent vagal $\mathrm{C}$ fibers arising from the left ventricle, finally resulting in bradycardia. The clinical response is varied with a majority of the patients exhibiting a mixed response and the remainder either a cardioinhibitory or vasodepressor response (3). The term "malignant neurocardiogenic syncope" is used to refer to a more serious form of the disorder. In Europe (4) the term "malignant" has been used to address neurocardiogenic syncope that occurs without warning symptoms and results in physical injury. In North America (5) the term is reserved for neurocardiogenic syncope that results in profound cardioinhibition and asystole.

Stimulation of afferent vagal nerves can occur from either the heart or from structures including the great vessels (carotid bulb), eyes (oculocardiac reflex) and viscera including the gastrointestinal tract and urinary bladder (6). Pain due to mesenteric stretching and colonic distension can trigger increases in vagal tone. Often reaction accompanies intubation of a tightly looped sigmoid colon which results in tension on the sigmoid mesentery stimulating vagal response (6). The incidence of vasovagal reaction associated with colonoscopy has been reported to range from $0.1 \%$ to $1.3 \%$ in various series (7). Various triggers for such reactions including anxiety, the sight of blood or body tissues, visceral pain and distension and a warm crowded environment have been described. Isolated hypotension during colonoscopy may be attributed to dehydration and drugs such as narcotics and benzodiazepines. Whereas, a bradycardic response is likely to be mediated by vagal stimulation; it responds to withdrawal of the colonoscope. Herman et al. (8) showed that $16.5 \%(37 / 223)$ of patients experienced a vasovagal reaction during colonoscopy. Of these 37 patients, $35 \%$ required medical intervention in the form of intravenous fluids in 9 , and atropine and naloxone in 2 each. Though pain due to mesenteric distension has been implicated as the trigger by many authors, interestingly the present patient did not experience significant pain or discomfort during the procedure. Only one case report of a vasovagal reaction during rectosigmoidoscopy with a 28-second asystole has been published (9). The prolonged duration of asystole in the present patient with neurocardiogenic syncope shows that not all patients with neurocardiogenic syncope are the same and there is a subset of patients in whom the cardioinhibition can be profound enough to cause a life threatening event.

The value of an asystolic response on tilt testing has been a matter of debate. Milstein et al. (10) showed that all 6 survivors of asystolic sudden cardiac arrest with a normal electrophysiological study had a positive tilt response with severe bradycardia and hypotension with syncope in all six. Another large cohort of patients with a tilt-induced asystolic response showed no cardiac-related deaths (11). But the issue study (12) which used an implantable loop recorder to document events during syncope, has shown that the most common response in the "real world" scenario is a bradycardiac response, again reiterating the value of an asystolic response obtained on ambulatory recordings in these patients.

The line of demarcation between a vasovagal syndrome and sudden death may sometimes be thin. As proposed by Engel (13), in the face of emotional or physical stress the body reacts by raising the fright-flight and conservationwithdrawal response. In a healthy person the end result of this response is neurocardiogenic syncope whereas in patients with a susceptible heart or an abnormal conduction system this might result in sudden death. When a patient collapses but quickly recovers it is called fainting. When he 
dies in the next few minutes it is called sudden death. However, not all physicians may concur with the above statements. The present patient had a positive tilt test and a real life episode which was similar to the tilt response. Thus, it is imperative that to recognize the patient with a NCS who would be having a "malignant" form of this condition.

In conclusion, it would be prudent to monitor the cardiac rhythm of patients with a history of neurocardiogenic syncope who undergo medical procedures. Premedicating these susceptible patients with atropine, maintaining adequate hydration and minimizing pain may prevent a vasovagal response. These measures could render the procedure more comfortable and safe for the patient.

The authors state that they have no Conflict of Interest (COI).

\section{References}

1. Moya A, Sutton R, Ammirati F, et al. Guidelines for the diagnosis and management of syncope (version 2009). Eur Heart J 30: 2631-2671, 2009.

2. Screening for colorectal cancer. U.S. Preventive Services Task Force recommendation statement. Ann Intern Med 149: 627-637, 2008.

3. Grubb BP, Olshansky B. Syncope: Mechanisms and Management. Blackwell Futura, Malden, MA, 2005.
4. Fitzpatrick A, Theodorakis G, Vardas P, et al. The incidence of malignant vasovagal syndrome in patients with recurrent syncope. Eur Heart J 12: 389-394, 1991.

5. Grubb BP, Temesy-Armos P, Moore J, et al. Head-upright tilttable testing in evaluation and management of the malignant vasovagal syndrome. Am J Cardiol 69: 904-908, 1992.

6. Chen-Scarabelli C, Scarabelli TM. Neurocardiogenic syncope. BMJ 329: 336-341, 2004.

7. Eckardt VF, Kanzler G, Schmitt T, Eckardt AJ, Bernhard G. Complications and adverse effects of colonoscopy with selective sedation. Gastrointest Endosc 49: 560-565, 1999.

8. Herman LL, Kurtz RC, McKee KJ, et al. Risk factors associated with vasovagal reactions during colonoscopy. Gastrointest Endosc 39: 388-391, 1993.

9. Selleger C, Adamec R, Morabia A, Zimmermann M. Vasovagal syncope during rectosigmoidoscopy: report of a case. Pacing Clin Electrophysiol 11: 346-348, 1988.

10. Milstein S, Buetikofer J, Lesser J, et al. Cardiac asystole: a manifestation of neurally mediated hypotension-bradycardia. J Am Coll Cardiol 14: 1626-1632, 1989.

11. Barón-Esquivias G, Pedrote A, Cayuela A, et al. Long-term outcome of patients with asystole induced by head-up tilt test. Eur Heart J 23: 483-489, 2002.

12. Moya A, Brignole M, Menozzi $C$, et al. Mechanism of syncope in patients with isolated syncope and in patients with tilt-positive syncope. Circulation 104: 1261-1267, 2001.

13. Engel GL. Psychologic stress, vasodepressor (vasovagal) syncope, and sudden death. Ann Intern Med 89: 403-412, 1978.

(C) 2012 The Japanese Society of Internal Medicine http://www.naika.or.jp/imindex.html 